\title{
A Model of Support for Families of Children With Autism Living in the COVID-19 Lockdown: Lessons From Italy
}

\author{
Francesca degli Espinosa ${ }^{1,2}\left(\mathbb{D} \cdot\right.$ Alma Metko $^{3} \cdot$ Marta Raimondi $^{3} \cdot$ Michele Impenna $^{4} \cdot$ Elena Scognamiglio $^{5}$
}

Published online: 2 June 2020

(C) Association for Behavior Analysis International 2020

\begin{abstract}
Italy has been the European country most affected by the COVID-19 pandemic to date and has been in social lockdown for the longest period of time compared to other countries outside China. Almost overnight, Italian behavior analysts were faced with the challenge of setting up remotely whole-family systems aimed at maintaining adaptive skills and low levels of challenging behavior to be carried out solely by caregivers. Given these extraordinary circumstances, the protocols available from the applied behavioranalytic, parent training, and autism literature did not appear to fully meet the needs of parents having to be with their children under extreme levels of stress in a confined space with limited reinforcers for $24 \mathrm{hr}$ a day, 7 days a week. To meet this unprecedented challenge, we developed a dynamic and holistic protocol that extended to the full day and that recognized the need for sustainable intervention delivered solely by parents, who were often looking after more than one child. These practices are presented in this article, together with a discussion of lessons we have learned thus far, which may be useful for behavior analysts working in other regions in which the effects of the pandemic are not yet fully realized. Although somewhat unorthodox, we include some parent comments at the end with the goal of sharing the parent perspective in real time as this pandemic unfolds across the world.
\end{abstract}

Keywords Autism $\cdot$ Parent training $\cdot$ Telehealth $\cdot$ Token economy

\section{Editor's Note}

This manuscript is being published on a highly expedited basis, as part of a series of emergency publications designed to help practitioners of applied behavior analysis take immediate action to adjust to and mitigate the COVID-19 crisis. This article was submitted on April 5, 2020, and received final acceptance on April 10, 2020. The journal would like to especially thank Julie Kornack and Courtney Tarbox for their expeditious reviews of the manuscript. It is important to note that this article reports the approach taken by a particular group of clinicians operating under completely unprecedented circumstances in one of the hardest hit regions of the world. There are many ways to use the science of applied behavior analysis to support families, and neither the authors nor the journal suggests this is the only approach or the best approach. However, this approach produced positive results for this group of families, and the editorial staff at the journal believes that the rest of the world of applied behavior analysis may benefit from learning from their experience. The views and strategies suggested by the articles in this series do not represent the positions of the Association for Behavior Analysis International or Springer Nature.

Francesca degli Espinosa

degliespinosa@gmail.com

1 ABA Clinic, London, UK

2 University of Salerno, Fisciano, Italy

3 Private Practice, Bergamo, Italy

4 Private Practice, Rome, Italy

5 Private Practice, Naples, Italy
Before discussing responses to the pandemic, it may be helpful to say a few words about the Italian health system as it concerns intervention for autism based on applied behavior analysis (ABA). Italy has a national health system in which autism is recognized as a condition that falls under the care of the state. Education is free for all, and mainstream schooling is mandatory and therefore accessible by all children. There are no specialist schools. The delivery of therapeutic and educational services is regulated by registered professional bodies (i.e., psychologists, professional educators, speech and language therapists, neuro-rehabilitation technicians). ABA-based intervention is not formally recognized by the Italian health authorities, nor is it routinely offered as part of the state autism provision, which typically includes $1 \mathrm{hr}$ per week of psychomotor therapy, $1 \mathrm{hr}$ per week of logotherapy (i.e., speech and language therapy), and school attendance with varying levels of one-to-one educational support. The profession of behavior analysts is not officially regulated. Despite the lack of formal governmental recognition of $\mathrm{ABA}$ interventions for autism and a corresponding professional body, the country has witnessed a steady increase in the number of professionals credentialed by the Behavior Analyst Certification Board (BACB) in the past 10 years, verified course sequences, and state-funded health 
rehabilitation centers offering low-intensity ( 4 to $15 \mathrm{hr}$ per week) ABA intervention. As a result of parental demand for ABA services, a considerable number of health professionals are enrolling in ABA master's programs and consequently incorporating ABA-based methods in their therapeutic practices for autism. Schools have also begun to open their doors to BACB-credentialed professionals to support the individualized education plans of their students. Nevertheless, ABAbased intervention remains largely privately funded by individual families and is, for the most part, carried out at home during the hours in which the child is not in school. At the time of this writing, the Italian authorities have yet to provide statewide guidelines or funding for the continuation of intervention (ABA and non-ABA) via telehealth for children with autism during the lockdown period. In some regions (e.g., Campania, Lombardy, Marche, Emilia Romagna), and with considerable variability, individual state-funded centers have begun to set up systems to provide intervention and parental support remotely.

\section{The Present Crisis}

Italy was one of the first European countries, together with Germany, France, and Spain, to register the first cases of COVID-19 at the end of January 2020 and subsequently to impose movement restrictions on its citizens. For some weeks, it was the second country after China with the largest number of COVID-19 cases. Presently surpassed by the United States and Spain, it currently registers the highest number of deaths related to COVID-19 (World Health Organization, 2020). On January 31,2020 , the Italian government declared a state of national emergency and imposed the first social-distancing restrictions on February 24, with a decree closing all schools and many commercial activities in the northern regions (Lombardy and Veneto). These restrictions were gradually expanded and eventually extended to the rest of the country, with the period of complete national lockdown commencing on March 9 (Ministry of Health, 2020). At the time of writing this paper, Italy continues to be in complete lockdown, which includes home isolation, with outside movement restricted to one person per household and solely for the purpose of purchasing food or medicine. Deliveries to households are limited to essential goods. Outside physical activity is no longer permitted. However, if a child has a disability, she or he can be accompanied outside for brief walks, provided the parent carries a written certificate signed by a health professional attesting to the child's diagnosis and need to be outside.

Social-distancing measures during the initial lockdown period were less restrictive and were expected to last for a couple of weeks. Although children had ceased going to school, and most home sessions had been interrupted, ABA practitioners viewed this as a period similar to the summer holidays, where children spend long periods of time with grandparents and are essentially given free access to reinforcement, and parents are given a skeleton program of maintenance to prevent significant skill loss. Under usual circumstances, we would expect to see some increase in challenging behavior and some skill loss during the summer months, but not so significant that it could not be addressed within the first few weeks of resuming the typical school and home intervention schedule.

By the third week of the lockdown, it became clear that the isolation period would not only be extended to the rest of the country, but that measures would also become much more restrictive. Thus, a different approach was required, especially because many parents (all family caregivers in the home are referred to as "parents" hereafter, for brevity) reported that their children were no longer satisfied with the usual reinforcers; were becoming increasingly uncooperative; were engaging in high levels of stereotypy and problem behavior, likely due to being denied access to regular but now unavailable reinforcers (e.g., swimming, going to the playground, taking extended car rides, going to the cinema or ice-cream parlor, participating in physical activity); were demanding high levels of undivided attention; and were becoming more difficult to direct to independent activities.

Parents also reported that they were struggling to reconcile the demands of distant working required by their employers with the needs of round-the-clock sole care of their children with autism, of siblings, and of their households in the absence of any outside help. Although some families lived in the countryside and had access to a privately owned garden (i.e., private yard), many families lived in city apartments, where time spent outside was either prohibited or substantially limited due to the closure of parks and the shared courtyards. Even those of us who are not psychologists saw clear early signs of mental health decline, as well as increasing marital conflict. Although the latter problems were outside the scope of our practice as behavior analysts who are not also psychologists, referral to paid online psychotherapy or counseling, given the dire financial situation some families were experiencing, was not an option. Nonetheless, we believed that the tools of the science of behavior could be extended to the larger family context to alter the repertoires of all its members and to increase contact with positive reinforcement for all.

Because restrictions in Italy happened gradually, with families in the northern regions being in the first cohort, by the time families in the southern region were in lockdown, we had gathered sufficient data on the effects of the first 3 weeks of isolation with minimal structure and free reinforcement access. Whereas for the northern families our work in the third week was focused on reducing the negative effects of 2 weeks of "free time," we were able to implement strategies proactively with the southern families to minimize the negative outcomes we observed for the families in the first lockdown cohort. 
Prior to the lockdown period, none of our children displayed severe or unmanageable levels of challenging behavior, and they all had effective behavior management plans in place. After the first 2 weeks of lockdown with limited structure and free reinforcement access, in some of our first cohort families we observed the following during our online meetings: high levels of escape from simple instructions, the loss of independence and communication skills (appropriate mands), satiation (significant reduction in the time children spent with favorite items), an increase in problematic interactions between parents and all children, and unmanageable levels of mands for attention (both appropriate and inappropriate). Parents reported being struggling to find new things to entertain their children with autism and siblings. Sourcing novel toys or items to create new interests was not possible due to limited deliveries of nonessential items.

In the following sections, we describe the protocol that was shaped through the frequent interactions with parents during our online observations and discussions. Currently, as a group of professionals, we are serving approximately 30 families with this model. We were consulting with these families prior to lockdown and had been running home-based programs for at least 6 months. In the absence of published literature on interventions that require parents to engage with their children $24 \mathrm{hr}$ per day, 7 days a week, in a confined space for a prolonged and undefined time period, we approached the problem inductively, altering what we did based on principles of learning, on what each individual situation required, and on what parents reported they felt they were able to do. The model developed was a systemic one, in which the client was no longer just the child in sessions working on educational targets but, rather, the whole family in its unique context.

\section{Risk Assessment}

We began with an assessment of risk and an evaluation of each child's level of verbal functioning to establish the type of telehealth provision required (direct sessions or parent coaching; see Ferguson, Craig, \& Dounavi, 2019, for a review). The two main risks we aimed to mitigate were (a) parental burnout and (b) an increase in socially mediated challenging behavior of the child and siblings. We were less concerned with behavior maintained by automatic reinforcement, unless it was self-injurious. None of our children engaged in such behavior. The assessment was based on our history with the family, data from the child's prelockdown intervention, and direct contingency manipulation and observation via telehealth. For example, we asked the parent to leave his or her child with an activity while he or she talked to us, and we calculated how long the child was engaged without demanding parental attention.
The main items included in the risk assessment (see Table 1) were parents' prelockdown level of instructional control and social engagement with their children, the duration of the child's and siblings' ability to engage in solitary activities (either reinforcement or instructional, e.g., worksheets, chores, functional play), and parents' tolerance of nondangerous selfstimulation (e.g., flapping, noises, walking up and down, jumping on the sofa). In addition, we considered the child's age, the ability to engage in back-and-forth verbal interaction, the presence of siblings with a disability, and the number of supportive adults always present (i.e., single parent, both parents at home, other family members).

For each individual family, we assessed the risk as high, medium, or low. The highest risk families were ones with a single parent or a parent with limited instructional control, who was on his or her own most of the day with two young children, one of whom had autism. In the case of one family,

Table 1 Risk assessment interview and observation form.

\begin{tabular}{|c|c|c|c|c|}
\hline $\mathrm{N}$ & Questions & Respc & onses & Comments \\
\hline 1 & Is the parent a single parent? & Yes & No & \\
\hline 2 & Is the parent alone most of the day? & Yes & No & \\
\hline 3 & $\begin{array}{l}\text { Is one of the two parents working from } \\
\text { home? }\end{array}$ & Yes & No & \\
\hline 4 & Are both parents working from home? & Yes & No & \\
\hline 5 & $\begin{array}{l}\text { Are there other supportive adults living in } \\
\text { the household? Who? }\end{array}$ & Yes & No & \\
\hline 6 & Are there siblings? How many? & Yes & No & \\
\hline 7 & Does the sibling have a disability? & Yes & No & \\
\hline 8 & Is the sibling attending online schooling? & Yes & No & \\
\hline 9 & Is the sibling an infant or toddler? & Yes & No & \\
\hline 10 & $\begin{array}{l}\text { Can the sibling engage in solitary activities } \\
\text { without demanding parental attention for } \\
\text { one hour or longer? }\end{array}$ & Yes & No & \\
\hline 11 & For half an hour? & Yes & No & \\
\hline 12 & Is the child 2 to 5 years of age? & Yes & No & \\
\hline 13 & $\begin{array}{l}\text { Would the child be able to engage in direct } \\
\text { sessions online? }\end{array}$ & Yes & No & \\
\hline 14 & $\begin{array}{l}\text { Does the child need constant supervision } \\
\text { and instruction? }\end{array}$ & Yes & No & \\
\hline 15 & $\begin{array}{l}\text { Can the parent tolerate not intervening if } \\
\text { the child engages in non-dangerous } \\
\text { levels of self-stimulation? }\end{array}$ & Yes & No & \\
\hline 16 & $\begin{array}{l}\text { How long can the child spend by himself } \\
\text { without requiring parental intervention } \\
\text { (e.g., self-stimulation or tangible } \\
\text { reinforcer) }\end{array}$ & Yes & No & \\
\hline 17 & $\begin{array}{l}\text { Can the child engage in an independent } \\
\text { appropriate activity for } 30 \text { minutes } \\
\text { without adult supervision? }\end{array}$ & Yes & No & \\
\hline 18 & For 15 minutes? & Yes & No & \\
\hline 19 & $\begin{array}{l}\text { Is the parent able to interrupt the child from } \\
\text { a reinforcing activity without problem } \\
\text { behaviour? }\end{array}$ & Yes & No & \\
\hline 20 & $\begin{array}{l}\text { Does the child cooperate with simple } \\
\text { instructions without problem behaviour? }\end{array}$ & Yes & No & \\
\hline
\end{tabular}


the parent was alone for most of the day with two adolescents with autism, one who was minimally verbal and the other who was verbally interactive. Although this situation could have been considered high risk, in this specific case we considered it medium risk because the parent had excellent instructional control over both youngsters, who were both able to engage in solitary activities. As a general rule, the higher the risk, the higher the level of support we provided for the parent and the greater the daily structure.

Low-risk families included ones with both parents at home who were willing to engage with the child and sibling. In some cases, parents took turns to be with both children, so that one parent could be free. Alternatively, each parent looked after one child, and they swapped children every few hours or every day. In these cases, it became crucial to ensure that both parents were involved in the care of the child and sibling, so that the burden of household management did not fall solely on the primary caregiver (Parent 1). To achieve the involvement of both parents, provided they were both equally available, we worked separately with each one, setting up individual targets with each and developing a family schedule in which the time of each member was clearly specified.

\section{Level of Verbal Functioning}

We identified three main learner profiles of the children, in terms of their need for support and perceived amenability to direct sessions over telehealth:

1. preschool-age children $(n=6)$ : children who had not yet started elementary school (up to age 7);

2. minimally verbal children: children with limited adaptive, independent, and verbal skills $(n=16)$; and

3. verbally interactive children $(n=8)$.

Together with the risk assessment, this classification determined the daily family structure we arranged, as well as the type and frequency of support we provided as professionals.

Verbally interactive children were defined as being able to discriminate $W h-$ questions (tact and intraverbal), follow multiple-step instructions, self-administer tokens, selfmanage interruption of reinforcement based on a timer, and not manifest challenging behavior in and out of sessions. Verbally interactive children were those who we predicted would be able to sustain a direct session with their ABA tutor (also commonly referred to as a technician or therapist) via telehealth. Although none of the tutors had ever delivered interventions in this manner, extensive training was not needed because of their experience with the program targets and their familiarity with the child's home environment. For these families and tutors, contact with the ABA consultant (commonly referred to as a supervisor) and/or lead tutor occurred once or twice per week to review targets, ensure that novel problem behavior was not emerging, and continue to provide support to the parents in managing the day.

Verbally interactive children received sessions in two formats. The first format involved the tutor remotely sharing the computer screen with the child, so what was once the table became the desktop computer. All visual stimuli were placed in individual electronic folders or PowerPoint presentations, and the child responded to the materials presented via the tutor's desktop. Tutors did not hold up cards to the screen, as it was too cumbersome, and some tutors also did not have the relevant materials at home. The second format was implemented with adolescents who were working on producing written responses; in that case, it was the child who remotely shared his or her screen with the tutor. The data collection system for these children remained unaltered from the prelockdown period, as the only change was the medium of delivery. Most children received two 50-min sessions per day.

For the two remaining profiles, preschool age and minimally verbal, we implemented a parent coaching system (see Parsons, Cordier, Vaz, \& Lee, 2017, for a review). The first telehealth session lasted up to $3 \mathrm{hr}$ and was conducted by the team's ABA consultant, with the participation of both parents and the team's lead tutor. Subsequently, the consultant or lead tutor met with the family every day during the first and second week and every other day during the third intervention week and thereafter. The following protocol applies to families with preschool-age or minimally verbal children. The youngest child was 4 years old and had been receiving intervention for approximately six months. Most of our families had been running an ABA home-based program with the support of an ABA consultant and tutor(s) for a minimum of 2 years and some for as long as 10 years.

Parents were not asked to take data, as this seemed unrealistic given how demanding their day was. Professionals collected data during the online coaching sessions on the following:

- parental report of challenging behavior;

- parental report of their ability to maintain the agreed structure;

- direct measurements of children's adherence to parental instructions;

- direct measurements of challenging behavior during the coaching session; and

- parents' procedural fidelity.

\section{Parent Coaching System}

Prior to the lockdown period, children had access to a range of environments, each associated with its unique set of stimuli, signaling a specific reinforcement contingency. For example, children had learned that at school or during home sessions, 
brief periods of reinforcement were provided contingent on engaging in instructional activities and exchanging tokens. Thus, children spent most of their learning time in school and in home sessions, where reinforcement access was regulated. For most of our children, the domestic context with parents signaled prolonged and often uninterrupted access to reinforcement in the evenings and on weekends. Although not ideal, prior to the lockdown period, we did not view this as a significant problem because in most of their daily contexts (e.g., school and home sessions), children were able to engage in educational activities appropriately. During lockdown, however, the household became the only living context for all family members. This new set of circumstances created the need to institute an economic system that was sustainable, easy to implement for parents, and positively reinforcing for all members. The system also needed to promote the maintenance of adaptive skills and positive interactions, as well as allow for time off from interaction.

\section{Structuring the Day}

During the first consultation session with each family, we worked with parents on structuring the entire day for all children (the child with autism and sibling), dividing it into blocks of activities to meet primary needs (breakfast, morning snack, morning outside time, lunch, afternoon nap for the younger children, afternoon snack, afternoon outside time, bath, and dinner). All times in between were considered dead times and, therefore, high-risk times that needed to be filled with contextually appropriate activities alternated with reinforcement intervals.

One important aspect of structuring the day was to decrease the number of waking hours to reduce the fatigue and behavioral irritability that occurred toward the latter part of the day. We achieved two periods of roughly comparable duration between the mornings and afternoons by pushing lunchtime from 12:00 to 13:30-14:00 and bringing bedtime forward to no later than 21:00. As is fairly typical of Southern European countries, our children often went to bed around 22:30 prior to the lockdown. We worked with parents to manipulate the stimuli associated with the end of the day (e.g., supper, bath, pajamas, story) to occur earlier than usual and in accordance with the recommendations for the optimal number of sleep hours for the child's chronological age (Hirshkowitz et al., 2015). Table 2 shows an example of a daily schedule for a family in which Parent 1 was alone most of the day with a 5-

Table 2 Example of a daily schedule of a parent alone most of the day with two young children.

\begin{tabular}{|c|c|c|c|c|}
\hline & Child & Sibling & Parent 1 & Parent 2 \\
\hline 08:00-08:30 & Wake up, washing, dressing & Wake up, washing, dressing & Child & Sibling \\
\hline 08:30-09:00 & Breakfast & Breakfast & Both children & Unavailable \\
\hline 09:00-09:30 & Adult-led activity & Adult-led activity with child or independent skill & Both children & Unavailable \\
\hline 09:30-10:00 & Reinforcement & Reinforcement & Free & Unavailable \\
\hline 10:00-10:30 & Adult-led activity & Adult-led activity with child or independent & Both children & Unavailable \\
\hline $10: 30-11: 00$ & Garden & Garden & Both children & Unavailable \\
\hline $11: 00-11: 30$ & Garden & Garden & Both children & Unavailable \\
\hline $11: 30-12: 00$ & Snack & Snack & Both children & Unavailable \\
\hline $12: 00-12: 30$ & Adult-led activity & Adult-led activity with child or independent skill & Both children & Unavailable \\
\hline $12: 30-13: 00$ & Reinforcement & Reinforcement & Free & Unavailable \\
\hline $13: 00-13: 30$ & Reinforcement & Meal preparation with parent 1 & Meal preparation with sibling & Unavailable \\
\hline $13: 30-14: 00$ & Lunch & Lunch & Child & Unavailable \\
\hline $14: 00-14: 30$ & Chores with parent & Nap & Child & Unavailable \\
\hline $14: 30-15: 00$ & Reinforcement & Nap & Free & Unavailable \\
\hline $15: 00-15: 30$ & Independent skill teaching & Nap & Child & Unavailable \\
\hline $15: 30-16: 00$ & Reinforcement & Activity with parent & Sibling & Unavailable \\
\hline $16: 30-17: 00$ & Snack & Snack & Both children & Unavailable \\
\hline $17: 00-17: 30$ & Walk & Garden & Supervision & Walk with child 1 \\
\hline $17: 30-18: 00$ & Walk & Reinforcement & Free & Walk with child 1 \\
\hline 18:00-18:30 & Reinforcement & Activity with parent 2 & Free & Activity with Sibling \\
\hline 18:30-19:00 & Activity with parent 2 & Reinforcement & Free & Activity with child \\
\hline 19:00-19:30 & Reinforcement & Meal preparation with parent & Meal preparation with sibling & Free \\
\hline $19: 30-20: 15$ & Meal & Meal & Meal & Meal \\
\hline $20: 15-21: 00$ & Bath, pyjamas, bedtime Parent 1 & Bath, pyjamas, bedtime Parent 2 & Child & Sibling \\
\hline
\end{tabular}


year-old child with autism with limited independent and selfentertainment skills and a 3-year-old typically developing sibling.

\section{Choosing Contextually Appropriate Activities}

The day consisted of a rotation of contextually relevant activities or tasks for both the child and the sibling. We asked parents to identify one target per day in any of the activities, 1-week objectives, and 3-month objectives. We formulated the questions in this way: What would be helpful for you that your child learned? What do you want to teach your child today? What do you want to have taught your child in 1 week? When all this is over, what would you want your child to be able to do? In 1 month? In 3 months? Parents chose from four types of activities broadly defined as follows:

1. Independent activity: This encompassed any instructional activity the child could engage in without adult support (e.g., puzzles and shape sorters, worksheets, coloring, educational computer programs, domestic skills). Visual activity schedules were utilized where useful (McClannahan \& Krantz, 1999).

2. Household chores: These included chores the parent felt he or she could carry out with his or her child, giving the child things to do. We asked parents to go to each room and list all the chores that needed doing in each, however big or small. We asked parents to list every chore and not just the chores they thought their child could do or was already able to do. We wanted to identify objectives that were appropriate to the context and in which the parent was more likely to engage their child, as they needed to be done anyway.

3. Tabletop discrete-trial teaching (DTT): We did not ask parents to run acquisition targets but only to maintain existing skills, with particular focus on clean responding without behavioral accessories (e.g., stereotypy). Although it would be desirable if children maintained specific skill targets in specific programs, our purpose for having parents run DTT was primarily to help ensure that the children maintained some contact with the DTT contingency of rapid and accurate responding.

4. Adult-led or shared activity (not DTT): These included activities that required the parent to engage one or both children, in which responses could be more loosely defined. Examples of shared activities were completing simple crafts or making cookies. These were not necessarily reinforcing activities for the child with autism but often were reinforcing for the sibling. Parents were coached to divide their attention between the two children, shape appropriate attention mands, and provide attention contingent on participation and engagement with the material. The targets for the child with autism were simply to remain in the activity and engage in some relevant responding.

We did not include activities based on natural environment teaching (NET), in which the parent actively had to manipulate the child's motivation and materials to evoke mands or to generalize language targets. This was because parents reported that they found this type of approach to be too effortful under these extreme circumstances. They reported they did not wish to be in a position to have to follow their child's motivation and to have to signal when that was no longer possible, risking the occurrence of challenging behavior. They also could not risk having to say no to something their child requested because of a lack of materials. Although, in general, the daily structure centered on adult-led or shared activities alternated with periods of solitary reinforcement, all parents learned to interact with their children and siblings in a way that worked for them and maintained low rates of problem behavior. It is important to note that previous research exists that supports remote training of parents in NET procedures (Nefdt, Koegel, Singer, \& Gerber, 2010), so we are not suggesting that this would not be a good approach for some families.

\section{Setting Up the Household's Positive Reinforcement System}

Two main reinforcement systems were implemented throughout children's waking hours: a token-based economy and an activity-based economy. In the token-based system, tokens were earned throughout the day and exchanged for preferred items. In the activity-based system, engagement in a less preferred activity (i.e., contextually appropriate activities) produced access to a more preferred activity. Whether token based or activity based, the common element in both procedures was a system in which contingent relations between target behavior and preferred objects and activities were maintained, and reinforcers were unavailable outside those particular settings. We aimed to help parents establish a closed economy, meaning that engaging with parent-led reinforcement contingencies was the only way in which children could access those reinforcers, which is known to generate higher levels of responding (Kodak, Lerman, \& Call, 2007; Reed, Niileksela, \& Kaplan, 2013). Because we had already witnessed in our northern families (first cohort) the negative impact of free and prolonged access to reinforcers on (i.e., loss of children's skills and parental fatigue), we organized the household economic system so that reinforcement was accessible contingent on the production of contextually appropriate behavior for all children. This included the siblings, if they were under the age of 10 and not involved in remote schooling. 
The first step in closing the economy involved teaching parents to be able to limit access to all reinforcers for the child and siblings in every room of the household. Parents classified reinforcers in terms of solitary and social for all children. Solitary reinforcers were those that the child and sibling could consume on their own, and social reinforcers were those that required an adult and, where possible, were incorporated in the shared and adult-led activities. To help parents identify effective reinforcers, we accompanied them virtually through each room of the house and asked them to identify things their child liked or might like or items with which they had witnessed their child spend some time. Every item was removed and placed in boxes, inside wardrobes or bags, or whatever container was available. In some cases, parents took pictures of the items to produce a reinforcer menu for the child (and sibling), whereas at other times, they took the child to the "shop," the place where all reinforcers had been stored. Food treats were not available from the shop or the reinforcer menu; these were available at specific times during meals. The reasoning behind this was that food treats were items to be consumed rapidly, so the child was engaged for a very short period of time.

Our rationale in discontinuing noncontingent access to solitary toys and activities was that these items would hold their value and keep the children occupied longer. If the children were therefore able to be engaged with preferred solitary activities, the parent could safely have time off from the child and sibling. The secondary effect was to reduce escapemaintained problem behavior for both the child and the parent. By engaging with children in structured activities first, parents could access time to themselves and, as a result of contingent reinforcement delivery, also gain greater instructional control. We wished to create a mutually reinforcing situation for both the adults and the children, where parents could experience success in delivering instructions and interacting with their child, given that they would need to engage in this behavior daily in a confined space, over extended periods of time without a break. Of course, discontinuing free access to reinforcers could also have the effect of increasing motivating operations that evoke problem behavior, so it was critical to support the children to be frequently successful in meeting the criteria for positive reinforcement, and thereby continuing to earn reinforcement on a frequent basis.

Many parents implemented the new reinforcement contingencies effectively, and the children learned very quickly that reinforcers were only available after the completion of activities or upon meeting the token schedule requirements. Access to the reinforcer menu or shop was not available at any other time. Children's mands for these items when they were not available were significantly reduced. Because there were clear signals for reinforcement availability, children stopped asking for these items at other times, preventing parents from having to say no, thus reducing the risk of challenging behavior. Children learned to mand for items (and receive them) only when the reinforcer menu was presented or when they were taken to the shop. They used whichever communication modality (e.g., vocal, sign, pointing, or selection-based modality) had been achieved prior to the lockdown period. Given the level of stress the parents were experiencing after several weeks of lockdown, the likelihood was extremely high that they would reinforce problem behavior by providing the denied item to interrupt contact with the aversive stimulation produced by the child. We wished to reduce the risk by implementing the simplest possible system and not burdening parents with having to teach their child to tolerate denial. We simply removed that risk by giving frequent but contingent access to those items.

\section{Token Reinforcement System}

The token system was implemented mainly for children who had limited verbal and nonverbal skills and who required frequent contact with reinforcement to engage in parent-led activities and instructions. A token system had been in place for all such children prior to the lockdown period, either at school or during home-based sessions. Because we had always worked at home, all parents were familiar with the basic techniques; in fact, it was generally parents who manufactured the token boards, so the concept of a token economy system was not new to them. The fact that the system needed to be extended to the entire day was new and, in this sense, paralleled early applications of comprehensive token economy systems (Ayllon \& Azrin, 1965, 1968; Phillips, 1968).

Our token schedules consisted of three interrelated components:

1. the token-production schedule (the schedule by which responses earn tokens);

2. the exchange-production schedule (the schedule by which exchange periods are earned); and

3. the token-exchange schedule (the schedule by which the tokens were cashed in for preferred items or activities; see Hackenberg, 2018, for a review).

The initial token-production schedule was set at fixed ratio (FR) 1, in which each target response (carrying out the parent's instruction within the activity proposed) produced a token to produce rapid acquisition. This was implemented throughout the day each time the parent gave an instruction. Fairly quickly, parents naturally moved to a variable ratio 
schedule, in which a variable number of responses was required to produce a token, and they learned to adjust it based on the time of day or the difficulty of the task.

The exchange-production schedule was fixed at FR 10, in which 10 tokens were needed to reach an exchange period. We did not make this variable, as it would have been too difficult for the parents to manage. The token-exchange schedule was FR 1-handing over the token boardwhereupon the parent presented the reinforcer menu or took the child to the shop to choose one item.

During the exchange period, only one item or activity was allowed at a time. If the child wished to change the activity, then tokens had to be earned again for the change to occur. Reinforcement duration varied according to parental needs and what was established by the daily schedule. In general, there were two types of reinforcement duration: brief (between 1 and $5 \mathrm{~min}$ ) and long (up to $30 \mathrm{~min}$ ). If parents needed their child to be occupied for additional time, they still had to interrupt after 30 min of consumption, place the item back in the shop, run a quick token board, and then open the shop or present the reinforcement menu again.

\section{Activity-Based Reinforcement System}

The activity-based system was implemented when the parent had more than one child to look after, and it was extended to all siblings under the age of 10 if they were not involved in remote schooling. The system was based on creating half-hour blocks in which the parent was coached to engage the child and sibling in an adult-led or independent instructional activity for $30 \mathrm{~min}$ in order to produce $30 \mathrm{~min}$ of reinforcement time for everyone (the child, the sibling, and the parent).

\section{Lessons Learned}

In the present article, we have described a model of supporting Italian families during the past 6 weeks of lockdown. As professionals, we realized that this was one of the greatest challenges we would face in our careers. It soon became apparent, however, that the published literature and other tools upon which we typically rely were insufficient to deal with the magnitude and urgency of the present crisis. Substantial previous research had been published on aspects of ABA service delivery via telehealth, but little or no previous research had evaluated systems for transferring entire ABA programs from in person to telehealth overnight, especially in the context of families living under total lockdown. In the absence of specific guidelines, we relied on an inductive process dictated by the tradition of our science, adapted published protocols, and derived procedures from principles. The system we were called to develop needed to be comprehensive and efficient. It needed to recognize the complexity of each individual family dynamic and be, at the same time, simple, realistic, and sustainable to maintain parental engagement.

Some may find the nearly complete elimination of noncontingent reinforcement and the application of a token economy across all waking hours to be somewhat extreme. However, the primary problem reported to us by parents before making this change was a lack of structure and loss of child motivation due to continuous free access to reinforcers. By programming reinforcement contingent on active engagement with the household schedule, we empowered the parents to increase their child's motivation and provide clear direction for everyone involved. If the parents had not been effective in providing sufficient antecedent support in the form of prompting and setting task difficulty at an achievable level, then such a system could have resulted in inadequate access to positive reinforcement. However, with support from their ABA consultants, parents were successful in bringing order to their homes and helping their children to be calm, productive, engaged, and happy.

Despite the difficulties we are all experiencing, as both professionals and human beings, we have learned some valuable lessons that we hope will shape our ability to serve our families more effectively in the future. We have tremendous respect for the courage and dedication shown by the families we work with, who, at a time of adversity and uncertain future, have remained focused on the present. Although parents realize that we are all learning as we go, we have seen a level of parental engagement that we had not been able to generate previously. Such change, although borne out of crisis, may have enduring positive effects. Our task moving forward will be to maintain these novel repertoires under more positive contingencies.

\section{A Closing Note From Parents}

We have not yet been able to analyze the data so far collected. We have included ongoing assessment of social validity by asking parents to comment on their experiences so far. We present three representative translated excerpts of parents' feedback:

I thought it would be difficult to maintain the daily schedule of alternating instruction with reinforcement, but it has been very successful. I have stuck to it, and it has been all very natural and not too effortful. I am also very happy because I am able to spend time with my 2year-old, who is also making progress. I am able to play more with him and to focus on his speech. It's going well. (Vittoria, parent of B, a 6-year-old girl with autism, and G, a 2-year-old boy) 
We received very simple and clear instructions - take away all reinforcers, engage him all day in simple domestic chores, give him routines, and give the reinforcers only after completing a token board. We saw an immediate change, zero problem behavior, and collaboration from C. If this situation had not happened, my husband and I would never have had such an enriched experience. Seeing C so calm and compliant is the biggest reinforcer. (Giada and Davide, parents of $\mathrm{C}$, a 9-year-old boy with autism)

The management of $\mathrm{G}$ became very difficult. All his routines and perception of time had been disrupted. G, who was never interested in playing, became satiated with technology and was constantly searching for food, becoming very anxious during mealtimes. Creating a closed reinforcer economy and dividing the day in clear sequential moments as to not get to the point of acute problem behavior and prevent boredom were essential. As parents, even in the absence of tutors, we are able to manage our child calmly and maintain learned skills. (Veronica and Giorgio, parents of G, a 7-year-old boy with autism)

\section{Compliance with Ethical Standards}

Conflict of Interest The authors declare that they have no conflicts of interest.

Ethical Approval This article does not contain research conducted with human subjects.

\section{References}

Ayllon, T., \& Azrin, N. H. (1965). The measurement and reinforcement of behavior of psychotics. Journal of the Experimental Analysis of Behavior, 8(6), 357-383. https://doi.org/10.1901/jeab.1965.8-357.
Ayllon, T., \& Azrin, N. H. (1968). The token economy: A motivational system for therapy and rehabilitation. New York, NY: AppletonCentury-Crofts.

Ferguson, J., Craig, E. A., \& Dounavi, K. (2019). Telehealth as a model for providing behaviour analytic interventions to individuals with autism spectrum disorder: A systematic review. Journal of Autism and Developmental Disorders, 49(2), 582-616. https://doi.org/10. 1007/s10803-018-3724-5.

Hackenberg, T. D. (2018). Token reinforcement: Translational research and application. Journal of Applied Behavior Analysis, 51(2), 393435. https://doi.org/10.1002/jaba.439.

Hirshkowitz, M., Whiton, K., Albert, S. M., Alessi, C., Bruni, O., DonCarlos, L., et al. (2015). National Sleep Foundation's updated sleep duration recommendations: Final report. Sleep Health, 1(4), 233-243. https://doi.org/10.1016/J.SLEH.2015.10.004.

Kodak, T., Lerman, D. C., \& Call, N. (2007). Evaluating the influence of postsession reinforcement on choice of reinforcers. Journal of Applied Behavior Analysis, 40(3), 515-527. https://doi.org/10. 1901/jaba.2007.40-515.

McClannahan, L. E., \& Krantz, P. J. (1999). Activity schedules for children with autism: Teaching independent behavior. Bethesda, MD: Woodbine House.

Ministry of Health (2020). Coronavirus, le misure adottate dal Governo, provvedimenti attualmente vigenti, approvati dal Governo in seguito all'emergenza sanitaria internazionale. Retrieved April 5, 2020, from http://www.governo.it/it/approfondimento/coronavirus/13968

Nefdt, N., Koegel, R., Singer, G., \& Gerber, M. (2010). The use of a selfdirected learning program to provide introductory training in pivotal response treatment to parents of children with autism. Journal of Positive Behavior Interventions, 12(1), 23-32. https://doi.org/10. 1177/1098300709334796.

Parsons, D., Cordier, R., Vaz, S., \& Lee, H. C. (2017). Parent-mediated intervention training delivered remotely for children with autism spectrum disorder living outside of urban areas: Systematic review. Journal of Medical Internet Research, 19(8), e198. https://doi.org/ 10.2196/jmir.6651.

Phillips, E. L. (1968). Achievement place: token reinforcement procedures in a home-style rehabilitation setting for "pre-delinquent" boys. Journal of Applied Behavior Analysis, 1(3), 1311003. https://doi.org/10.1901/jaba.1968.1-213.

Reed, D. D., Niileksela, C. R., \& Kaplan, B. A. (2013). Behavioral economics. Behavior Analysis in Practice, 6(1), 34-54. https://doi.org/ 10.1007/BF03391790.

World Health Organization. (2020). Coronavirus disease (COVID-19) pandemic. Retrieved April 5, 2020, from https://www.who.int/ emergencies/diseases/novel-coronavirus-2019.

Publisher's Note Springer Nature remains neutral with regard to jurisdictional claims in published maps and institutional affiliations. 